\title{
Keine Ahnung: Über etcetera-Formeln und Verwandtes
}

\author{
Wie, wenn es verboten wäre zu sagen „Ich weiß“ und erlaubt nur zu sagen \\ „Ich glaube zu wissen“? (Wittgenstein 1984: Über Gewißheit, §366)
}

\section{Gesprächs-Führung}

Es ist nicht genug zu wissen, was man sagen will und es dann auch hinreichend kohärent zu äußern. Den eigenen Gedanken zu folgen und ein kommunikatives Ziel im Auge zu behalten, ist das eine, wie man das erreicht, ein anderes. Kohärenz und Zielstrebigkeit des eigenen Tuns beizubehalten, ist in der vielfältigen Verflechtung alltäglichen Sprechens zwar auch schon eine nicht zu unterschätzende Aufgabe. Stehen die Sprechenden doch immer in der Gefahr, von etwas, was eigentlich ein struktureller Nebengedanke hätte sein sollen, davongetragen $\mathrm{zu}$ werden und in einer ganz anderen Geschichte anzukommen. Aber das ist eine andere Geschichte - vielleicht aber auch doch nicht so ganz. So mancher Nebengedanke findet seinen Weg in die Rede, und mehr Platz als ihm ursprünglich zugedacht war, weil man denkt, man müsse noch etwas an stützender Information nachlegen, um die gewünschte Information an die Partnerinnen und Partner zu bringen, sie den eigentlichen Hauptgedanken verstehen zu lassen. Wir reden ja nicht für uns selbst - und selbst da dialogisieren wir oft einmal -, wir wollen und müssen das, was wir sagen, nicht nur darauf abstimmen, was wir direkt sagen wollen, um den Beteiligten die Information zukommen zu lassen, die wir gerne vermitteln würden. Es bedarf vielmehr des Bedenkens von allerlei MetaBedingungen. Wir müssen dann, was wir sagen, darauf abstimmen, was die Partner erfahren sollen und was sie - vermutlich - schon wissen oder es aus verschiedenen Gründen erschließen können. Zudem muss die Abstimmung auf solche Erwartungen und die Modifikation der Planungen aufgrund von Reaktionen der Partner „on the fly“ erfolgen, im Fortgang der Rede, in dauernder Rückkopplung mit den erkennbaren und erwartbaren Erwartungen des Gegenübers. Dass Dinge erwartbar sind, beruht auf der Annahme und Existenz geteilter Wissensbestände und sprachlicher Handlungsmuster als Praktiken der Interaktion. So ist der reine Text des Gesprochenen häufig nur ein Gerüst dessen, was da geschieht. Auf der anderen Seite gehören verlässliche Konventionen, wenn man so will, Sprach- 
spiele, $\mathrm{zu}$ den wegweisenden und die Erwartungen steuernden Elementen in Interaktionen. ${ }^{1}$

Die relative Verlässlichkeit der Konventionen, die sich in wiedererkennbaren Sprachspielen niederschlagen, bildet den Rahmen für eine ökonomische Handhabung der Anforderungen an ein „gutes“ Gespräch, das durch die immerwährende Nachjustierung der interaktionellen Wege gekennzeichnet ist. Das zeigt sich an vielen Erscheinungen, die beim Fortgang und der Führung eines Gespräches eine Rolle spielen. ${ }^{2}$

\section{Annahmen und Erwartungen}

Wenn wir mit jemandem über etwas (oder auch über jemanden) sprechen, geht es also daher nicht ohne Annahmen ab, die dann in diesem Kontext gerne Vorannahmen heißen, und die Sprechenden, aber vielleicht mehr noch die jeweils Hörenden, bringen auch Erwartungen in ein Gespräch ein. Und diese Erwartungen lassen sich in gewissem Sinn als Umkehrung der in Karl Bühlers OrganonModell entfalteten Funktionen verstehen. Wobei die Ausdrucksfunktion aus dieser Gegensicht den Annahmen und Erwartungen auf der Beziehungsebene entspricht. Das ist ganz offenkundig, zwingt uns doch schon der Beginn eines Gesprächs zu mancherlei Entscheidungen. Der Beginn eines Gesprächs kennt einige durchaus formalisierte Schritte, die uns auf dieser Ebene Entscheidungen abverlangen, etwa schon bei der Wahl einer Grußformel. Sie soll ja eine akzeptable nähere, fernere oder neutrale Distanz herstellen, und auch etwas über den intendierten Formalitätsgrad sagen. Hier ist in den letzten Jahrzehnten einiges in Bewegung geraten, und so ist es nicht verwunderlich, dass unter dem Einfluss veränderter Verhältnisse in der Sprechsprachlichkeit des Deutschen zum Beispiel sozial wenig differenzierende und eher kolloquiale Grußformeln wie etwa hallo an Bedeutung gewinnen. Zudem, da sie noch nichts im Hinblick auf die Anredepronomina - das Duzen bzw. Siezen - präjudizieren. Die Appellfunktion betrifft in dieser Umkehrung der Sicht die Frage der jeweiligen kommunikativen Hierarchie, in gewisser Weise die Angemessenheitsebene, was sich zum Beispiel in Fragen der sprachlichen Üblichkeiten im Kontext verschiedener Praktiken und der

1 Dass sie sprachlich angekündigt und in erwartbaren Formen reproduziert werden, zeigen auch die Möglichkeiten, sie elektronisch in Dokumentationen von Interaktion wiederzufinden; vgl. z.B. die Beispielanalyse des Typs „Trostdialog“ in Scharloth (2016: 328-332).

2 Zu den in Kapitel 1 nur angedeuteten Phänomenen siehe inhaltlich Eichinger (2017); dort wird auch auf die einschlägige Literatur verwiesen. 
darin eingenommenen Rollen niederschlägt. Dabei dienen zum Beispiel salvatorische Einleitungsformeln vom Typ ich glaub auch dazu, mögliche Rollenkonflikte abzufedern.

Uns soll es hier aber um die Entsprechung der dritten der Bühler'schen Funktionen gehen, um die Darstellungsfunktion. Aus der Sicht einer gesprächsbegleitenden Strategie, in der Annahmen und Erwartungen über die adäquate Weise der Informationsvermittlung zu klären sind, geht es um die Fragen einer angemessenen Informationsstrukturierung und um Strategien der Annäherung an eine adäquate Informationstiefe.

\subsection{Informationsvermittlung}

Was den ersten Punkt angeht, den der Informationsstrukturierung, so wird beim Sprechen eine Vielzahl von Hinweisen auf folgende und abgeschlossene Äußerungen gegeben, zum Teil wird auch ein internes Innehalten signalisiert. Einen neueren Klassiker, der sich letztlich alle diese funktionalen Optionen erobert hat, stellt im Deutschen der Internationalismus ok dar, ${ }^{3}$ dem zum Beispiel auch die Verwendung von genau in gewissem Umfang zu folgen scheint. Solche Elemente sind in gewissem Maß die kommunikativen Begleiterscheinungen der allmählichen Artikulation der sich fortentwickelnden Gedanken. Es ist ja auch so, dass sie zum Teil ein räsonierendes Sprechen mit sich selbst begleiten. Man kann das an den verschiedenen Funktionen der Partikel genau ganz gut sehen.

aber ich bin in ja genau glaub in vier oder in fünf wochen bin ich ne woche in stuttgart (FOLK_E_00066_SE_01_T_0,182, IDS-Datenbank DGD) ${ }^{4}$

3 Eine Übersicht dazu findet sich in Nilsson (2013: 154-162).

4 Die Belege in diesem Beitrag stammen aus drei Korpora des Instituts für Deutsche Sprache (www1.ids-mannheim.de/onlineangebote), und zwar 1) dem Deutschen Referenzkorpus (DEREKo) mit Texten der geschriebenen Gegenwartssprache (www.ids-mannheim.de/cosmas2), aus dem bestimmte Zeitungstexte („Interviews“ u.ä.) und Wikipedia-Diskussionen herangezogen werden; 2) aus der Datenbank für Gesprochenes Deutsch (DGD), über die ausgewählte Korpora des Archivs für Gesprochenes Deutsch zugänglich sind (http://dgd.ids-mannheim.de/dgd/ pragdb.dgd_extern.welcome), insbesondere das Teilkorpus FOLK (http://agd.ids-mannheim.de/ folk.shtml), das Gesprächsdaten aus unterschiedlichsten Bereichen enthält; 3) aus dem Korpus „Deutsch heute“ (http://prowiki.ids-mannheim.de/bin/view/AADG/AnHang), das aus systematisch erhobenen Sprachaufnahmen (freie Interviews und evozierte Daten) von Oberstufen- und Volkshochschülern (letztere ca. 45 bis 60 Jahre alt) aus dem gesamten deutschen Sprachraum besteht. 
(2) S1: weil da jetzt / minus zwei steht ist das jetzt die zweite binomi// binomische formel // S2: richtig genau wei hier minus is // S1: ach so // S2: genau (FOLK_E_00274_SE_01_T_0 562-569, IDS-Datenbank DGD)

(3) S1: immer // mit ix wert anfangen // S2: ja immer mit ix wert anfangen / S1: okay // S2: genau immer (FOLK_E_00274_SE_01_T_0, 123-128, IDS-Datenbank DGD)

Im Beleg (1) geht es bei genau - eigentlich der Kombination ja genau - um eine selbstreflektive Vergewisserung über die folgende Aussage, in (2) um den positiv resümierenden Abschluss einer (handlungsführenden) Interaktion und in (3) um die zustimmende Einleitung zu einer bestätigenden minimalen Äußerungseinheit - wobei hier die zwischengeschaltete positive Antwortpartikel okay beiläufiger wirkt, als es ein weiteres genau wäre. Im folgenden Beispiel sieht man, wie ein Element wie okay äußerungsinitial und äußerungsfinal genutzt werden kann, also zu Ankündigung und Abschluss, so dass es sich eigentlich um ein kontextuell jeweils auf seine Weise zu interpretierendes Grenzsignal handelt:

(4) S1: okay // jetzt kommt en neues thema he he // S2: welche aufgabe machen wir // S3: // hm // S1: jetzt machen wir // seite elf nummer eins // S2: okay (FOLK_E_00274_SE_01_T_0,385-393, IDS-Datenbank DGD)

Es ist an allen diesen Beispielen erkennbar, dass im fortlaufenden Erzeugen von Äußerungen sehr viel häufiger die Notwendigkeit besteht und die Gelegenheit gesucht wird, Aussageelemente und -abschnitte kenntlich zu machen und so den Partnern das Folgen zu erleichtern.

\subsection{Informationstiefe und Explizitheit}

Etwas anderes ist es, wenn die Passung der übermittelten oder zu übermittelnden Information abgeschätzt und die Art und Dichte der Information darauf abgestellt werden soll. Die Antworten fallen in diesem Fall sicher bei gesprochener Sprache deutlich anders aus als bei geschriebener Sprache. Tendenziell sind die

Bei jedem Beleg werden neben der Korpusquelle ggf. Informationen zur Einordnung des Belegs (z.B. zum Texttyp, zum Thema oder zu den Informanten) gegeben. Die Transkription wurde auf die für den gegebenen Zusammenhang notwendigen Informationen reduziert; Pausen werden durch Schrägstriche / bzw. // markiert, Sprecherwechsel durch die Personenkürzel (das Kürzel $S 1$ steht für Sprecher 1 etc.). 
Strategien zu einer möglichst ökonomischen Handhabung dieses Problems in beiden medialen Formen genau entgegengesetzt. Natürlich wird man in beiden Fällen versuchen, sich am vermuteten Informationsniveau der Adressierten zu orientieren, aber dennoch ist im Zweifelsfall unterschiedlich vorzugehen.

Wenn man nicht nachfragen kann, was bei strikter Schriftlichkeit als Normalfall anzunehmen ist, muss die Wahl dahin gehen, so explizit wie möglich zu sein - und andererseits trotzdem Redundanz zu vermeiden. Man muss die Dinge nicht zweimal schreiben, wenn sie ohnehin nochmals nachgelesen werden können. Dass bei dem damit verbundenen Bemühen um möglichste Eindeutigkeit die Gefahr droht, dass die Genauigkeit als Pedanterie gelesen wird, ist offenkundig. Ein gutes Beispiel dafür sind wissenschaftliche Texte, die von der Mitte her zu lesen gar nicht so einfach sind, da nicht, wie im praktischen sprachlichen Leben, aus den Umgebungsbedingungen Informationen und Begründungen geholt werden können.

In diesem Sinn ist das folgende Zitat aus Robert B. Brandoms opus magnum „Expressive Vernunft“ einerseits sowohl ein Beleg für schriftsprachliche Explizitheit, wie es andererseits inhaltlich einen Kommentar zu den differenten Anforderungen praktischen und praktischen sprachlichen Handelns darstellt:

Propositional gehaltvolle Festlegungen sind zunächst einmal solche, die Gründe abgeben und für die welche benötigt werden können; indem Festlegungen und die sie ausdrückenden Performanzen eine angemessene Rolle in den Praktiken des Gebens und Verlangens von Gründen spielen, wird ihnen propositionaler Gehalt verliehen. (Brandom 2000: 400)

Für das zielgerichtete Tun des Menschen eines ebenso praktischen wie sprachlichen Wesens, um die Umgebung von Brandoms zitierter Stelle andeutungsweise zu zitieren, ist so die praktische Umgebung eine Art Regulativ für die notwendige oder verlangte Explizitheit dessen, was als gültiger Grund für eine Festlegung anerkannt wird. Damit reduziert sich bzw. es muss im Einzelfall verrechnet werden, was als hinreichend explizit gelten kann, um eine Äußerung als im Sprachspiel berechtigt (,richtig“) anzusehen bzw. dem Äußernden die Berechtigung zuzuerkennen, die Äußerung als akzeptierten Zug in das Spiel einzubringen. Das hat im alltäglichen Sprechen logischerweise zur Folge, dass Techniken gesucht werden, die die Grenze des Hinreichenden „austesten“. Insofern es um argumentativ entlastende, verkürzende Techniken geht, ist Konventionalität eine vernünftige Strategie, um in der diskursiven Kontoführung ${ }^{5}$ als gültig aner-

5 Vgl. Brandom (2000: 402). 
kannt zu werden. Im Praktischen mag es allerdings auch darum gehen, wie Konventionalisierung funktioniert.

In diesem Kontext kann man eine Reihe von Fügungen und Redewendungen verstehen, die auf den ersten Blick wie die Verweigerung der Übernahme kommunikativer Verantwortung aussehen, sich aber bei einem zweiten Blick als eine generelle Anapher von als hinreichend angesehener Spezifizität bzw. Allgemeinheit verstehen lassen. Es sind das jene Dinge, die unter anderem unter dem Terminus etcetera-Formeln geführt werden.

Wenn man von der Abstraktheit der ganz generellen Funktion einer ökonomischen Handhabung von Begründungspflichten in praktischen Gesprächen zurücktritt, kann man bestimmte Akzentuierungen feststellen, und vielleicht auch so etwas wie uneigentliche Redeweisen. Wenn man sich ansieht, welche Formen unter diesem Aspekt betrachtet worden sind, so ist ihr konventioneller Charakter relativ klar. Gängige Formen dieser Vagheitsmarker mit unterschiedlicher Funktion sind Formeln, bei denen mittels der Konnektoren und und oder ein die Unsicherheit markierendes Element angeschlossen wird, prototypisch dafür sind und so bzw. oder so.

Wenn man Gesprächstypen betrachtet, in denen solche Fortführungen wahrscheinlich sind, sieht man erstens eine Vielfalt der Verwendungen und eine Bandbreite der Variation dieser Muster - mit einer signifikanten Ausdifferenzierung der gewählten Varianten. Wahrscheinlich sind solche Fortführungen zweifellos in nicht $\mathrm{zu}$ formellen Frage-Antwort-Kontexten, wie sie zum Beispiel im Korpus „Deutsch heute“ des IDS in großer Zahl vorliegen. Bei dieser Erhebung wurden verschiedene Arten von gesprochener Sprache elizitiert bzw. dokumentiert, darunter eben auch Interviews: Wenn man diese Interviews auf solche Verwendungen hin betrachtet, kommt man zu den folgenden Befunden. Es findet sich erstens eine ganze Menge solcher Fügungen, nicht ganz 4800 Belege für und so und über 7000 Verwendungen von oder so. Die folgenden Beispiele zeigen, dass sie auf unterschiedliche Kontexte und formale und semantische Kategorien bezogen sein können:

(5) ja an sich würde ich schon gerne hier bleiben // weil Familie und so das ist mir dann schon wichtig (Oberstufenschülerin aus Bielefeld, IDS-Korpus „Deutsch heute“)

(6) ja also ein bisschen weil meine Mutter die // hat es mal gelernt richtig und dann so im Urlaub machen wir ein bisschen // so ein kleines bisschen üben oder was und so (Oberstufenschüler aus Bad Waldsee, IDS-Korpus „Deutsch heute“) 
(7) für mich wäre immer ein Traum vielleicht irgendwann mal ins Ausland Richtung Kanada oder so weil da eben // [...] da ist immer noch ein bisschen mehr Natur wie bei uns in Deutschland (Oberstufenschüler aus Schrobenhausen, IDS-Korpus „Deutsch heute“)

(8) [Großstädte] zum einen ist es klar also sie sind nicht ich will jetzt nicht sagen hässlich oder so aber sie sind // unübersichtlich (Oberstufenschüler aus Siegen, IDS-Korpus „Deutsch heute“)

\section{Vorläufiges}

\subsection{Weiteres: und so (weiter)}

Wie angedeutet, macht die Verbindung aus dem prototypischen additiven Konnektor und mit der adverbialen Partikel so den Löwenanteil der Belege aus. Die entsprechenden Verwendungen von so werden im Paul'schen Wörterbuch (2002: 919) unter der Bedeutungsgruppe 3.1 klassifiziert, die folgendermaßen beschrieben wird:

auf die dem Hörer/Leser bekannte, im Gesagten näher bezeichnete, vorgeführte o. durch die Umstände deutlich werdende Weise, zumeist anstelle einer genauere Angaben enthaltenden adv. Bestimmung.

Die Verwendung in der Fügung und so ist eine Verwendung, die unmittelbar an andere grammatikalisierte Vorkommen von so anschließt. ${ }^{6} \mathrm{Zu}$ denken ist an Verwendungen mit Indefinita, also im Singular mit so ein bzw. im Plural lediglich mit so. ${ }^{7}$ In all diesen Fällen und auch in dem vorliegenden kann man diese Partikel als eine Anweisung verstehen, nach weiteren Elementen einer Art zu suchen. Schön kann man das an dem folgenden Ausschnitt aus dem längeren Beleg (11) sehen, der auf eine Art Party-Kontext verweist:

(9) erzählen un gucken und so un trinken un essen un so

Es wird durch das erste und so ein ergänzender Slot für kommunikative Tätigkeiten aufgemacht, mit dem zweiten einer für ,gesellschaftliche Nahrungsauf-

6 Siehe Duden (2016: §§119, 132).

7 Siehe Knöbl (2014). 
nahme“. Man kann sich die Szene additiv ausgestalten. Und tatsächlich geht die sprechende Person auch davon aus, dass hiermit für die Hörenden eine hinreichende Informationstiefe erreicht wird - übrigens ganz unabhängig davon, wie weit diese nun tatsächlich weitere Optionen zur Füllung der angebotenen Lücken beitragen könnten. ${ }^{8}$

An formalen und funktionalen Varianten finden sich beim und-Typ ansonsten die im Folgenden kurz charakterisierten Gruppen. Im Wesentlichen handelt es sich um drei Muster, und zwar erstens Bildungen mit dem Element weiter, zum anderen um Erweiterungen durch die quantifizierenden pronominalen Elemente alles und (irgend)etwas. Als dritten, etwas andersartigen, Typ kann man die reine (normalerweise dreifache) Iteration des und verstehen. ${ }^{9}$

Nicht überraschend ist, dass und so weiter, die feste und auch schriftsprachlich gängige Form und fast schon Ausbuchstabierung der Kurzform usw., die häufigste sonstige Variante darstellt; sie gehört ja zum Inventar fester lexikalischer Wendungen, wie die etwas stilistisch markiertere Variante mit und so fort. ${ }^{10}$ Zusammen sind sie nicht ganz 500-mal belegt.

(10) Robin, so weiß ich, arbeitet als Elektriker, er hat schwierige Lebensphasen hinter sich, Alkohol und so weiter, aber das ist vorbei (taz, 4.1.2016, IDSKorpus DeREKo)

(11) dann war ich einmal im theater war schön bei de premierenfeier dachte mir oh drei stunden theater is ja schön un mit_n bisschen und so weiter und so weiter ne erzählen un gucken und so un trinken un essen un so un dann komm ich hin / dann komm ich nach_er vera / anstaltung wieder komm ich wieder un / mein fahrrad is weg ey (FOLK_E_00148_SE_01_ T_020715, IDS-Datenbank DGD)

In ihrer Konventionalität scheint hier eigentlich nicht so sehr eine Konkretisierung erspart, ${ }^{11}$ vielmehr geht es um einen Verweis, dessen Vagheit eindeutig mit

8 Ich würde das damit etwas handlungs- und sprachspielbezogener fassen wollen, als das bei König/Stoltenberg (2013: 30) geschieht, wo es heißt: „Sprecher unterstellen also ein geteiltes Wissen bzw. ein intersubjektives Verständnis darüber, wie das bisher Gesagte in dem jeweiligen Kontext sinnvoll fortgesetzt werden kann.“

9 Siehe unten Belege (23) und (24).

10 Vgl. z.B. die Beschreibungen und Querverweise in den Einträgen zu weiter und so fort im Paul'schen Wörterbuch.

11 Belege vom folgenden Typ sollte man vermutlich gar nicht zu dieser formelhaften Verwendung rechnen: „Ein Korn Getreide auf dem ersten Feld eines Schachbretts, zwei auf dem zweiten, vier auf dem dritten, acht auf dem vierten und so weiter - immer doppelt so viel“ (SZ, 2.1.2016, 
einer gemeinsamen Kenntnis, einer Rekonstruierbarkeit mit dem Partner rechnet, auch wenn das, wie (11) zeigt, nicht endgültig sein muss.

In der längeren Form ist vielleicht sogar noch stärker die Unerheblichkeit der konkreten Ergänzung impliziert. ${ }^{12}$

(12) Ich weiß nicht, was sie in dem Moment gedacht hat, aber sie dürfte sich in allen Vorurteilen, die sie gegenüber Schrebergärtnern je hatte, bestätigt gefühlt haben: Sexisten, Patriarchen, Kleinbürger, Rassisten und so weiter und so fort (Zeit, 28.4.2016, IDS-Korpus DEREKo)

(13) ie ham äh d die ham // recht viel geld dann dort für die verhältnisse und so weiter und so fort ham spaß gehen in diskotheken und so weiter und so fort das wollen die auch (FOLK_E_00042_SE_01_T_02, 32-36, IDSDatenbank DGD)

In dieser Hinsicht ist die zweithäufigste Variante relativ ähnlich, in der ja nur auf einen gesamthaften Rahmen verwiesen wird: Alles, was dazugehört (und alle ${ }^{\star}$ ); alle Untertypen zusammen sind ungefähr 300-mal belegt:

(14) die kriegen dann / erst wird gefragt wie die schmerzen sin wie stark sie sin dann wird erst mal blutdruck und alles gemessen un dann gibt_s / schmerzmittel (FOLK_E_00228_SE_01_T_0, 1949, IDS-Datenbank DGD)

(15) es is doch okay die is doch auch offen und alles und die hat dann / meiner / schwester en vortrag darüber gehalten / wie wichtig es doch sei zu heiraten (FOLK_E_00220_SE_01_T_04, 0123, IDS-Datenbank DGD)

(16) hatten wir doch $\mathrm{n}$ familienfest hier bruder / und f kinder / und allem meine schwester / mit anhang und und und wir hier un so weiter (FOLK_E_00161_SE_01_T_0, 0846, IDS-Datenbank DGD)

Das dritthäufigste Muster bezieht sich in der Formulierung auf die Salienz bestimmter Eigenschaften, wobei das in der sprechsprachlichen Form mit dem verkürzten Pronomen offenbar gegenüber einem generellen Hinweis auf Analoges zurücktritt, entsprechende Formen wie und so (et)was/irgendwas treten ca. 320-mal auf:

IDS-Korpus DeReKo). „Das Muttertier vererbt seine Vorlieben dem Kind weiter, das Kind seinem Kinde und so weiter und so fort“ (NZZ, 4.3.2016, IDS-Korpus DEREKo).

12 Diese Form scheint somit ein typischer Fall für die erste der bei König/Stoltenberg (2013: 30) angeführten Funktionen zu sein (s.o.). 
(17) im Winter noch Snowboarden und so was in der Woche halt (Oberstufenschüler aus Annaberg-Buchholz, IDS-Korpus „Deutsch heute“)

(18) S1: [...] dann machen se den reißverschluss auf und // S2: so eklig // S1: zischen ersch mal / zusammen en bier und sowas des find ich immer // S3: ja des hass ich ja echt (FOLK_E_00055_SE_01_T_0 0334-0338, IDSDatenbank DGD)

Erkennbar ist die schriftsprachnähere und formal explizitere Variante mit etwas eigentlich von einem anderen Typ, mit einer stärkeren Akzentuierung der „Art und Weise“, als eine Art verkürzender Generalisierung expliziter Wendungen vom Typ so etwas wie.

(19) Was ist dann zu tun? Dann muss man seine Geschichte wirklich sehr gut aufarbeiten, eine Therapie machen, sich einer Gruppe anschließen und so etwas. (Nordkurier, 4.2.2016, IDS-Korpus DEREKo)

Die konstruktionell ausführlicheren erweiterten Varianten haben mit dem Allquantor und dem indefiniten Pronomen Elemente, die auf eine in der einen oder anderen Weise zu bestimmende Beliebigkeit des Verweises schließen lassen, was durchaus in der Regel als ein Element der Geringschätzung gelesen werden kann - und in der Nähe entsprechender noch expliziterer Formeln steht, und all so was, und irgendwas o.ä.:

(20) ein nicht unwesentlicher Teil der damaligen Friedensbewegung war überzeugt davon, dass man, bevor man den äußeren (politischen) Frieden erreichen könne, man zuerst den inneren Frieden mit selbst finden müsse - und all sowas (Wikipedia; Diskussion: Neue Soziale Bewegungen, 29.10.2011)

(21) also so spätestens ab vierzehn fünfzehn geht_s auch echt mit psychosen und all so em kram los (FOLK_E_00287_SE_01_T_01, 0674, IDS-Datenbank DGD)

(22) und irgendwann war der druck dann zu groß sich da ständig an und abzumelden und irgendwas (FOLK_E_00066_SE_01_T_01, 0904, IDS-Datenbank DGD)

Auffällig ist, dass sich die Fügungen mit und vielfältiger entfalten als die im Folgenden noch zu besprechenden mit oder. Das dürfte damit zu tun haben, dass in der Konjunktion und als dem „Universalkonjunktor“ (Breindl/Volodina/Waßner 
2014: 408) die mögliche Iteration schon angelegt ist, die sich, wie angedeutet, in unterschiedlicher Weise als abschließendes oder ergänzungsheischendes Element verstehen lässt. Besonders deutlich ist das natürlich, wenn nun tatsächlich iteriert wird, also beim Typ und und und:

\begin{abstract}
Äußerungsabschließend kann ein iteriertes und die Funktion von und so weiter übernehmen: der Sprecher zeigt damit an, dass er seine eigene Äußerung als unvollständig verstanden wissen will und dass ein Bestandteil der Proposition mit einer typgleichen Fortsetzung ergänzt werden soll. Durch die steigernd wirkende Iteration und dadurch, dass der Sprecher die Fortsetzung nicht selbst gibt, signalisiert er gleichzeitig, dass er die zu ergänzenden Inhalte nicht aufzählen kann, weil es sich um so viele handelt, oder dass er sie nicht aufzählen will, weil er sie für irrelevant hält. (Breindl/Volodina/Waßner 2014: 425)
\end{abstract}

Tatsächlich ist es so, dass die dreiteilige Reihung in ihrer rhetorischen Wirkung sehr stark die Unaufzählbarkeit signalisiert - gerade auch, wenn sie schriftsprachlich genutzt wird, wie in den im Handbuch der Konnektoren zitierten Belegen.

In unseren sprechsprachlichen Belegen aus dem Korpus „Deutsch heute“ ist diese Iteration mit etwa einhundert Belegen immerhin auch bei den vier häufigsten Varianten der Nutzung von und in diesen Offenheitskontexten. Wie die folgenden Belege zeigen, findet sich dieser Typ aber auch in schriftlichen mit sprechsprachlicher Anmutung:

(23) Er muss ein gutes Training anbieten, er muss motivieren können, er muss mit jungen und alten Spielern gleichermaßen umgehen können, muss am besten auch noch die Wünsche des Vorstands umsetzen und und und (RZ, 5.1.2016, IDS-Korpus DeREKo)

(24) Beim Übertritt von El Salvador nach Honduras zum Beispiel waren wir erst nach zweieinhalb Stunden offiziell eingereist und mussten zwischendurch 27 Kopien anfertigen lassen, von Zahlungsbelegen, Pässen und und und ... (RZ, 12.1.2016, IDS-Korpus DeReKo)

Dieser Iterationstyp hat - wie weitere hier als marginal beiseitegelassene Fälle wie und bla, bla, bla o.ä. - stark rhetorischen Charakter, zweifellos mit einer gewissen Neigung zur Emphase, ${ }^{13}$ aber zweifellos nicht nur, eher fungiert seine Verwendung auch als Hinweis auf Endlosigkeit oder Beliebigkeit, auch im damit verbundenen Bruch der syntaktischen Struktur. Es spiegelt den Tatbestand, dass

13 Siehe dazu König/Stoltenberg (2013: 30). 
eigentlich keine vernünftige Füllung so vieler adjungierter Glieder denkbar ist. Die weitere Verbeliebigung durch die drei Punkte in (24) kann man auch als Korrelat von ungeduldiger Genervtheit lesen.

\section{2 Ähnliches: oder (irgendwie) so}

Der Weg vom und zum oder ist in gewisser Weise der von einer Reihe einschlägiger Instanzen hin zu einer Wahl plausibler Alternativen, also zu etwas, was mit Analogien und Ähnlichkeiten arbeitet. Und so betrifft die Charakteristik im Paul'schen Wörterbuch - obwohl beides genannt wird - eher das oder so: ${ }^{14}$

Die ugs. wohl aus der Verbindung mit ähnlich verkürzten, zugleich eine gewisse Unschärfe und Unsicherheit ausdrückenden oder so, und so 'und/oder ähnliche Dinge, Handlungen o. Ereignisse', verbunden mit dem Appell an den Gesprächspartner, den vorgegebenen Spielraum selbst inhaltlich weiter auszufüllen.

Bei den oder-Fügungen sind die grundlegenden Voraussetzungen schon etwas anders als bei der Fügung mit und - und sie entsprechen eher der gegebenen Ähnlichkeitsbeschreibung. Die Offenheit für Alternativen ist hier eigentlich schon angelegt, interessant ist lediglich, wie die Offenheit der Optionen manipuliert wird. Das gilt gerade auch im Vergleich mit und:

Es scheint ansonsten, dass der Gegensatz zwischen offenen oder geschlossenen Aufzählungen, Beispiellisten etc. keine Frage der semantischen Unterschiede innerhalb der Klasse der disjunktiven Konnektoren ist, vielmehr eher - in „Beispiel“-Zusammenhängen - durch den Kontrast und vs. oder ausgedrückt wird. Während additive Konnektoren primär geschlossene Listen anzeigen, sind disjunktive zunächst einmal Marker für offene Listen. (Breindl/ Volodina/Waßner 2014: 670)

Die Frage ist, wie diese Öffnung in solchen gebundenen Verwendungen genutzt wird, bei denen durch das so lediglich die Aufforderung gesetzt wird, eine passende Kategorie zu finden, über die sich eine Ähnlichkeitsbeziehung konstituieren lässt.

(25) aber wenn es jetzt der Arbeitsmarkt verlangt sage ich mal hätte ich auch keine Probleme nach was weiß ich Bochum oder so zu gehen (Oberstufenschüler aus Aschaffenburg, IDS-Korpus „Deutsch heute“)

14 Es wird dann auch nur ein oder-Beleg aufgeführt. 
(26) ich kann auch nicht Musik studieren weil ich dazu noch ein Begleitinstrument bräuchte also so Klavier oder so (Oberstufenschülerin aus Lüchow, IDS-Korpus „Deutsch heute“)

(27) wäre eigentlich so Medien oder so aber irgendwo im Hintergrund (Oberstufenschülerin aus Pasewalk, IDS-Korpus „Deutsch heute“)

Es ist nicht untypisch, dass in den Belegen der oder so-Formel noch weitere Vagheitsmarker auftauchen, die unter anderem auf den oben angedeuteten Zusammenhang der Verwendungen von so insgesamt verweisen. Es gibt allerdings auch in diesem Kontext Möglichkeiten, den Rahmen der Optionen einzugrenzen:

(28) Edeka-Markt oder so wo man einkaufen kann (Oberstufenschülerin aus Duisburg, IDS-Korpus „Deutsch heute“)

Man kann zudem feststellen, dass es sich bei oder so in einer großen Zahl von Fällen um eine stark lexikalisierte Formel handelt, die etwa bei Zeit-, Alters-, Größenangaben o.ä. eigentlich nur 'ungefähr' bedeutet so wie like im Englischen und genre im Französischen. ${ }^{15}$

(29) von hier nach München das ist vielleicht eine Stunde oder so (Oberstufenschüler aus Regensburg, IDS-Korpus „Deutsch heute“)

(30) man hat ja da äh eine Dreiviertelstunde glaub ich Pause oder so (Oberstufenschüler aus München, IDS-Korpus „Deutsch heute“)

Wie man in (30) sieht, kann das auch als eine nachgeschobene weitere Relativierung der Verantwortlichkeit gelesen werden; wobei in diesem Fall ja schon ein relativierendes glaub ich eingeschoben ist. ${ }^{16}$ In gewisser Weise handelt es sich um eine implizite Relativierung der eigenen Berechtigung der Äußerung bzw. damit eigentlich darum, die Verantwortlichkeit für die Gültigkeit der Aussagen von vorneherein abzumildern.

Im Fall des Musters oder so gibt es zwei wesentliche Variationstypen: Im einen Fall geht es um die Fokussierung dessen, was hier ähnlich sein kann, und zwar durch eine Art pronominaler und indefiniter Reduktion der oben erwähnten ad-substantivischen Verwendungen, und im anderen um eine Verstärkung der Wahlfreiheit bei den angedeuteten Alternativen durch die Hinzufügung indefini-

15 Siehe Rothe (2014).

16 Allgemeiner zu ich mein, ich glaub Knöbl (2011), Knöbl/Nimz (2013). 
ter pronominaler und adverbialer Verweise. Formal treffen beide Funktionen in der Form oder so was zusammen. Dabei sind Verwendungen wie in (31) oder (32) mit ihrem Bezug auf ‘so Berufe' bzw. 'so Äußerungen' eher von diesem kataphorischen ersten Typ, (33) repräsentiert - durch die Abtönungspartikel halt verstärkt - eher den generell indefiniten Typ 'so etwas (im Handlungskontext) Ähnliches':

(31) oder so Managerin oder so was (Oberstufenschülerin aus Cham, IDS-Korpus „Deutsch heute“)

(32) ja meine Mutter sagt auch cool oder so was (Oberstufenschüler aus Coesfeld, IDS-Korpus „Deutsch heute“)

(33) vom Hort abgeholt oder so was halt (Oberstufenschülerin aus Elsterwerda, IDS-Korpus „Deutsch heute“)

Allenfalls auch indefinit ist die „Ungefähr“-Verwendung wie im ersten Fall in (34) zu verstehen, während der zweite Fall in diesem Beleg die anaphorische Aufforderung gibt, im Handlungskontext „Zahnspange“ nach einer geeigneten Kategorie $\mathrm{zu}$ suchen, in der sich Alternativen $\mathrm{zu}$ den genannten (zentralen) Objekten („Drähte“) finden könnten - auch wenn man die vielleicht gar nicht benennen könnte.

(34) ne zahnspange // hatte ich auch mal hab ich aber schon seit nem jahr oder so was ni mehr // hm_hm auch äh nix übrig davon // also manchmal sind so drähte oder so was noch [drin] (FOLK_E_00129_SE_01_T_00111-0116, IDS-Datenbank DGD)

Aufgrund dieser funktionalen Vielseitigkeit ist es nicht überraschend, dass diese Fügung - und ihre etwas „konservativere“ Variante oder so etwas - mit etwa 550 Belegen die häufigsten Erweiterungsformen zum Muster oder so im Korpus „Deutsch heute“ darstellen. Der Kontext eines Zitats einer Lehrerin im folgenden Beleg macht den Charakter dieser Wendung deutlich.

(35) und wenn die da sagt wir haben einfach ein zu schlechtes Benehmen und sind nicht zuverlässig oder so etwas dann können wir nicht mit (Oberstufenschüler aus Chemnitz, IDS-Korpus „Deutsch heute“)

Eine nächste insgesamt größere Gruppe im Korpus „Deutsch heute“ stellen die Äußerungen dar, in denen ein Element mit \{irgend\} dazutritt: 
(36) und ich würde einfach gerne ein bisschen mithelfen oder so irgendwas (Oberstufenschüler aus Bregenz, Österreich, IDS-Korpus „Deutsch heute“)

(37) Tom Clancy oder irgend sowas weiß nicht also irgendwelchen (Oberstufenschüler aus Dresden, IDS-Korpus „Deutsch heute“)

(38) oder so irgendwie durch irgendwelche Regeln (Oberstufenschülerin aus Kiel, IDS-Korpus „Deutsch heute“)

(39) mal Bekannte trifft die irgendwo im Urlaub hier sind oder irgendwie sowas (Oberstufenschülerin aus Bergen, IDS-Korpus „Deutsch heute“)

Hier geschieht zweifellos nichts grundsätzlich Neues, auch ohne dieses Element wird die Suchanweisung nach möglichen alternativen Möglichkeiten ins Indefinite geöffnet. Allerdings deutet der auch formal sehr vage Anschluss der indefiniten Phrasen darauf, dass die Intention, vom Hörenden eine konkrete Auffüllung anzufordern, ganz deutlich zugunsten eines Verweises auf einen Kontext oder Hintergrund ausfällt, über dessen Vorhandensein hinaus nichts Konkreteres gesagt werden muss, der in diesem Sinn beliebig ist. Damit steht im Vordergrund, möglicherweise kritische Zugriffe auf das genannte konkrete Ereignis, Objekt usw. von vorneherein abzuwehren. Das ist wohl auch der Zusammenhang, der erklärt, warum diese Art der Relativierung als nicht besonders freundlich gelten kann.

In dieser Hinsicht anschließen lassen sich die rhetorischen Selbstbefragungen in satzförmiger Realisierung, von denen sich ungefähr 40 Belege im Korpus „Deutsch heute“ finden:

(40) S1: klar kann ich mir schon vorstellen mit_m bus ne / so un wenn ihr dann irgendwie aktionen habt in stadt_c und und und was weiß ich das fängt dann irgendwann erst nachmittags an / das // S2: ja aber dann kannste au nich hinkommen ne dat is dann schwierig // S1: das // aber ich sach mal / stadt_c ort_a geht ja noch / die leute die aus_m stadt_d oder was weiß ich noch kommen oder so (FOLK_E_00161_SE_01_T_0, 0411-0415, IDSDatenbank DGD) ${ }^{17}$

17 Zum Status dieser „ich weiß nicht“-Fügungen siehe Helmer/Reineke/Deppermann (2016) und Helmer/Deppermann (2017). 


\section{Endgültiges: keine Ahnung}

Es sieht in der Abfolge der bisher behandelten Formen, die dazu dienen, die angezielte Informationstiefe vage zu halten und Hinweise auf mögliche Konkretisierungen zu geben, so aus, als müsse man immer mehr Mittel nutzen, die vom Ungefähren reden, um möglichst endgültig aus der Verantwortung entlassen zu werden. Das betrifft dann nicht nur die an der entsprechenden Stelle nachgetragenen etcetera-Formeln der verschiedenen Arten, vielmehr sind sie Teil eines Modalisierungsinventars, das zusammenwirkt, um in abgestufter Weise die Gültigkeit von Aussagen und bzw. oder die Verantwortlichkeit des Äußernden zu relativieren. ${ }^{18}$ So können denn auch explizite Formen vom Typ 'nicht wissen' entsprechend genutzt werden, die wörtlich genommen die Geltung der Aussage zurücknehmen, wie oben schon in (40) oder auch in (41):

(41) den holen wir uns dann einfach Videothek oder was weiß ich (Oberstufenschüler aus Augsburg, IDS-Korpus „Deutsch heute“)

Sie stellen die konstruktionell expliziteste Möglichkeit in einem Kontinuum von Modifikatoren dar. So sieht man in den folgenden Belegen nicht nur die und so und die oder so Variante unserer bisher behandelten Formeln; diese werden kombiniert mit einfachen Verwendungen der Partikel so, mit Abtönungspartikeln, nicht zuletzt halt oder mit den geltungsmodifizierenden verbalen Konstruktionen ich glaub/mein o.ä.:

(42) oder also was ich / am meisten lese sind irgendwelche Bücher über Afghanistan oder / Iran oder so halt mit Taliban und so (Oberstufenschülerin aus Buchen, IDS-Korpus „Deutsch heute“)

(43) ich mein ich bin jetzt nicht so an an die Landschaft hier an die Region so gebunden irgendwie (Oberstufenschüler, aus Annaberg-Buchholz, IDSKorpus „Deutsch heute“)

Wie diese ich mein-Formeln haben auch Formen mit dem Verb wissen solche Verwendungen, die an Gesprächspartikeln erinnern:

18 Das erinnert an das, was man etwa zur Mehrfach-Negation im Mittelhochdeutschen sagt, nämlich dass hier insgesamt ein negativer Flair erzeugt werde, um dann den Satz negativ zu verstehen; vgl. die kurze Zusammenfassung in Weddige (2007: 75ff.). 
(44) wenn man viel redet / und halt immer dieses / Einschlag-R nimmt dann überschlägt man sich irgendwie weiß nicht dann geht das einfach nicht (Oberstufenschülerin aus Braunschweig, IDS-Korpus „Deutsch heute“)

Ein Beleg wie der Folgende zeigt, dass im Rahmen dieses Modalisierungsspiels die einzelnen formalen Elemente unterschiedlich eingesetzt werden können:

weil irgendwie also früher wo ich noch so in meiner weiß ich nicht revolutionären Phase war oder so weiß ich nicht genau mit vierzehn war ich schon so in so äh politischen Jugendgruppen auch gewesen und habe mich da war auch aktiv und so aber irgendwie habe ich dann hat es für mich keinen Sinn mehr ergeben (Oberstufenschülerin aus Frankfurt an der Oder, IDS-Korpus „Deutsch heute“)

In diesem Beleg - ähnlich wie auch oben schon in (25) - wird das zwischen Artikel und Adjektiv eingeschobene weiß ich nicht als eine Art Vorhalt benutzt, mittels dessen die fortdauernde Gültigkeit der impliziten Prädikation als 'revolutionär' relativiert wird: Ich weiß nicht, ob ich es noch so nennen soll. Dann kommt noch das relativierende oder so und dann noch einmal eine halb strukturierte weiß nicht-Fügung (ohne pronominale Flexion).

Die Steigerung von 'etwas nicht zu wissen' ist 'von etwas keine Ahnung zu haben', vielleicht sogar redensartlich 'nicht die blasseste Ahnung'. In dieser Bedeutung ist diese Wendung als umgangssprachliche Äußerungsoption seit dem späteren 19. Jahrhundert belegt. ${ }^{19}$ In den Interview-Teilen von „Deutsch heute“ ist diese Wendung so häufig belegt, dass sie offenbar - zumindest für die untersuchte Altersgruppe ${ }^{20}$ - fast als die Normalantwort dafür betrachtet werden kann, wenn man etwas nicht wisse. Insgesamt ist es dann so, dass von den etwa 1170 Belegen, die sich in dem Korpus finden, ca. 640 in Diskursfunktion, im Wesentlichen als Vagheitsmarker verwendet sind, ca. 380 als Antwortpartikel und der Rest von ca. 150 als „normale“ Prädikation. Wir wollen uns im Weiteren auf die Diskursfunktion beschränken. ${ }^{21}$

19 Siehe dazu Paul (2002) mit Verweis auf die Beleglage im Grimm’schen Wörterbuch.

20 Nach Auswertung der Daten des Korpus „Deutsch heute“ ist die Verwendung dieser Fügung bei den Oberschülern mehr als dreimal so hoch wie bei der Gruppe der älteren Gewährspersonen, die ebenfalls in diesem Korpus befragt wurden. Für die Auswertung des Materials danke ich Stefan Kleiner, Ralf Knöbl und den Hilfskräften des Projekts „Gesprochenes Deutsch“, Astrid Adler für die Unterstützung bei der Erarbeitung des Manuskripts.

21 Und aus Umfangsgründen zudem auf die Verwendungen am Äußerungsende, so dass manche Übergänge in den verschiedenen Verwendungen nicht sichtbar werden; eine Differenzie- 
Auffällig ist, dass diese Fügung sehr häufig im Kontext weiterer Vagheitsmarker auftaucht, signifikanterweise präferiert mit dem oder-Typ in verschiedenen Ausprägungen:

(46) also bei der komplexen Leistung sind es glaube ich fünfzehn Seiten die wir da schreiben müssen / und bei der besonderen Lernleistung sind es dann halt fünfzig oder so keine Ahnung (Oberstufenschülerin aus Chemnitz, IDS-Korpus „Deutsch heute“)

(47) ja ich denke mal schon wie bei öffentlichen Anlässen oder sowas keine Ahnung irgendwie wenn man eine Rede halten muss oder so (Oberstufenschüler aus Berlin, IDS-Korpus „Deutsch heute“)

Dazu kann man auch Kombinationen mit irgendwie rechnen, das in diesem Zusammenhang so etwas wie ein häufig auch nachgetragenes Satzadverb darstellt, also auch auf die Einschätzung des Sprechers Bezug nimmt:

(48) aber einfach nur so gerade weil ich gerade irgendwie Lust hatte keine Ahnung (Oberstufenschüler aus Pritzwalk, IDS-Korpus „Deutsch heute“)

In Anbetracht dessen, dass die Partikel halt eine Prätention auf geteilte Einschätzungen darstellt, ist sie auch ein geeigneter Begleiter für einen Konsens über die Nicht-Weiterverfolgung einer bestimmten Äußerungsspur, ${ }^{22}$ wie sie eine solche Verwendung von keine Ahnung nahelegt:

(49) und ich fand das halt irgendwie so keine Ahnung (Oberstufenschülerin aus Aachen, IDS-Korpus „Deutsch heute“)

Zum Teil auch nach mancherlei Wendungen der Modifizierung:

(50) ich weiß nicht das ist halt dann immer keine Ahnung / ich weiß nicht wenn sie halt irgendwie wenn wir gerade so rumscherzen dann ist es halt

rung der verschiedenen Verwendungsweisen aus gesprächsanalytischer Sicht findet sich in Bergmann (2017: 171-179).

22 Das mag z.T. vorläufig sein, und kann dann auch zu umgekehrten funktionalen Verteilungen von Elementen führen, vgl. das gegenseitige Verhältnis von keine Ahnung und halt im folgenden Beispiel: „naja so gut wie jeden Tag eigentlich also keine Ahnung am Abend halt vielleicht einmal eine halbe Stunde oder so“ (Oberstufenschüler aus St. Johann in Tirol, Österreich, IDSKorpus „Deutsch heute“). 
irgendwie uah keine Ahnung mit bairischem Einschlag auf jeden Fall (Oberstufenschülerin aus Furth, IDS-Korpus „Deutsch heute“) ${ }^{23}$

Es sieht so aus, als hätte sich mit dem, wenn man so will, verkürzten Nominalprädikat keine Ahnung ein Kandidat gefunden, der einigermaßen vielfältig einsetzbar ist, um eine Argumentation beiläufig, aber einigermaßen endgültig zu beenden. Ein Vorteil dieser Fügung scheint auch zu sein, dass sie - auch für jüngere Sprecher - kolloquial wirkt und gut in eine standardnahe Alltagssprache passt.

Das war's dann irgendwie so, keine Ahnung.

\section{Literatur}

Bergmann, Pia (2017): Gebrauchsprofile von weiß nich und keine ahnung im Gespräch - Ein Blick auf nicht-responsive Vorkommen. In: Blühdorn, Hardarik et al. (Hgg.): Diskursmarker im Deutschen. Reflexionen und Analysen. Göttingen: Verlag für Gesprächsforschung. 157-182.

Brandom, Robert B. (2000): Expressive Vernunft: Begründung, Repräsentation und diskursive Festlegung. Frankfurt a.M.: Suhrkamp.

Breindl, Eva/Volodina, Anna/Waßner, Ulrich Hermann (2014): Handbuch der deutschen Konnektoren 2: Semantik der deutschen Satzverknüpfer. 2 Teilbände. (= Schriften des Instituts für Deutsche Sprache 13). Berlin/Boston: De Gruyter.

Duden (2016): Duden 4: Die Grammatik. 9., vollst. überarb. u. aktual. Aufl. Berlin: Dudenverlag.

Eichinger, Ludwig M. (2017): Gesprochene Alltagssprache. In: Deutsche Akademie für Sprache und Dichtung/Union der deutschen Akademien der Wissenschaften (Hg.): Vielfalt und Einheit der deutschen Sprache. Zweiter Bericht zur Lage der deutschen Sprache. Tübingen: Stauffenburg.

Helmer, Henrike/Deppermann, Arnulf (2017): ICH WEIß NICHT zwischen Assertion und Diskursmarker: Verwendungsspektren eines Ausdrucks und Überlegungen zu Kriterien für Diskursmarker. In: Blühdorn, Hardarik et al. (Hgg.): Diskursmarker im Deutschen. Reflexionen und Analysen. Göttingen: Verlag für Gesprächsforschung. 131-156.

Helmer, Henrike/Reineke, Silke/Deppermann, Arnulf (2016): A range of uses of negative epistemic constructions in German: ICH WEIß NICHT as a resource for dispreferred actions. In: Journal of Pragmatics 106. 97-114.

Knöbl, Ralf (2011): Aspects of pluricentric German. In: Soares da Silva, Augusto/Torres, Amadeu/Gonçalves, Miguel (Hgg.): Línguas Pluricêntricas: Variação Linguística e

23 Im Hinblick auf die Verteilung voraus- bzw. rückwärtsweisender Verwendungen scheint die hier vorzufindende Reihenfolge typisch zu sein, zu den Zahlenverhältnissen siehe Bergmann (2017: 162 (mit 43 Belegen für keine Ahnung)). 
Dimensões Sociocognitivas. Braga, AXIOMA-Publicações da Faculdade de Filosofia. (https://ids-pub.bsz-bw.de/frontdoor/index/index/docld/4131, Stand: 30.11.17). 427-441.

Knöbl, Ralf (2014): Variation im Standard. Formale und funktionale Variationsaspekte des gesprochensprachlichen Gebrauchs indefiniter Referenzierungsformen. In: Bühler, Rudolf/Bürkle, Rebekka/Leonhard, Nina Kim (Hgg.): Sprachkultur - Regionalkultur. Neue Felder kulturwissenschaftlicher Dialektforschung. (https://ids-pub.bsz-bw.de/frontdoor/ index/index/docld/3240, Stand: 30.11.17). Tübingen: Tübinger Vereinigung für Volkskunde. 154-185.

Knöbl, Ralf/Nimz, Madlen (2013): Sprachräumliche Aspekte des Gebrauchs der deverbalen Modalpartikel glaub(e) zur Modulierung des Geltungsanspruchs von Äußerungen. In: Shamne, Nikolaj Leonidovich (Hg.): Raum in der Sprache. Raum der Sprache. Raum der Interaktionen. (https://ids-pub.bsz-bw.de/frontdoor/index/index/docld/1796, Stand: 30.11.17). Wolgograd: Verlag der Universität Wolgograd. 91-108.

König, Katharina/Stoltenberg, Benjamin (2013): „oder so“, „und so“, „und so was“, „und so weiter" etc. Eine interaktionale Perspektive auf Etcetera-Formeln. In: gidi Arbeitspapierreihe Nr. 48 (07/2013). (http://noam.uni-muenster.de/gidi/arbeitspapiere/ arbeitspapier48.pdf, Stand: 30.11.17).

Nilsson, Helena (2013): Zu Form- und Funktionsvariation der Gesprächspartikeln HM, JA, OKAY, und NEIN und ihren schwedischen Entsprechungen in der Chat-Kommunikation. (https:// gupea.ub.gu.se/bitstream/2077/32407/1/gupea_2077_32407_1.pdf, Stand: 30.11.17). Göteborg: Dissertation der Universität Göteborg.

Paul, Hermann (2002): Deutsches Wörterbuch. 10. Aufl. Tübingen: Niemeyer.

Rothe, Astrid (2014): genre - so ne Art französischer Diskursmarker. Über die Entwicklung des französischen Diskursmarkers genre am Beispiel von jugendsprachlichen Gesprächen. In: Weidenbusch, Waltraud (Hg.): Diskursmarker, Konnektoren, Modalwörter. (= Tübinger Beiträge zur Linguistik 522). Tübingen: Narr. 69-84.

Scharloth, Joachim (2016): Praktiken modellieren: Dialogmodellierung als Methode der interaktionalen Linguistik. In: Deppermann, Arnulf/Feilke, Helmuth/Linke, Angelika (Hgg.): Sprachliche und kommunikative Praktiken. (= Jahrbuch des Instituts für Deutsche Sprache 2015). Berlin/Boston: De Gruyter. 311-336.

Weddige, Hilkert (2007): Mittelhochdeutsch. 7. Aufl. München: C.H. Beck. Wittgenstein, Ludwig (1984): Über Gewißheit. Frankfurt a.M.: Suhrkamp. 